\title{
A Second Order Asymmetric Finite Difference Method for the Black-Scholes Equation of European Options
}

\author{
Wenbin Feng, Philsu Kim, Xiangfan Piao \\ Department of mathematics University of Kyungpook Daegu 702-701, Korea \\ fwbtown@naver.com, kimps@knu.ac.kr, piaoxf76@hanmail.net
}

\begin{abstract}
In this paper, we develop a fast numerical scheme for computing the European option pricing problems governed by the Black-Scholes equation. We prove that the proposed scheme has second order accuracy in both time and space. Under some restrictions, the stability of the proposed method in the sense of Von Neumann analysis is presented. It is shown that the proposed scheme has a good performance in the sense of the computational cost compare to the Crank-Nicolson scheme. Also the accuracy of the proposed scheme is better than the semi-implicit scheme in most cases.
\end{abstract}

Index Terms - Black-Scholes equation, European option pricing, asymmetric scheme, stability analysis, numerical example

\section{Introduction}

Black and Scholes (1973) firstly proposed an analytical formula for evaluating European call options value satisfying a lognormal diffusion partial differential equation which is now known as the celebrated Black-Scholes equation [1].

In option pricing problems, central difference secondorder finite differences (FDs) are commonly used for solving the Black-Scholes (B-S) equation. A recent work by Xiaozhong Yang and Lifei $\mathrm{Wu}$ [2] has focused on the use of the semi-implicit difference scheme for pricing vanilla options. The scheme is very efficient in the sense of the computational cost, but the semi-implicit difference scheme causes a low order rate (first order) of convergence for the time.

The aim of this paper is to improve the semi-implicit difference scheme. We propose a two-step asymmetric difference scheme which has the second order accuracy in both time and space. It is shown that the proposed asymmetric scheme has higher accuracy than the semi-implicit difference scheme in most cases. It is also seen that the computational cost of the asymmetric method is superior to the CrankNicolson scheme.

We begin the paper with the introduction of mathematical models for European call options. To avoid confusion, we will use the same notations in [2]. Assume that $\mathrm{V}$ is the call option value, $\mathrm{S}$ is the asset price, $\mathrm{t}$ is the time. Let $\mathrm{r}, \sigma$, $\mathrm{q}$ denote the risk-free interest rate, the volatility of the asset price and the continuous dividend yield, respectively. So the models considered in this paper are based on the BlackScholes partial differential equation [1]

$$
\frac{\partial V}{\partial t}+\frac{\sigma^{2}}{2} S^{2} \frac{\partial^{2} V}{\partial S^{2}}+(r-q) S \frac{\partial V}{\partial S}-r V=0
$$

where the explicit expression of B-S equation [1]:

$$
\mathrm{V}(\mathrm{S}, \mathrm{t})=\mathrm{S} e^{-q(T-t)} N\left(\mathrm{~d}_{1}\right)-K e^{-r(T-t)} N\left(\mathrm{~d}_{2}\right)
$$

The boundary condition of the Black-Scholes is given by:

$$
\mathrm{V}(\mathrm{S}, \mathrm{T})=\max \{\mathrm{S}-\mathrm{K}, 0\} \text {. }
$$

Introducing the change of variables

$$
\mathrm{x}=\ln S, \tau=\mathrm{T}-\mathrm{t},
$$

(1) can be rewritten as following

$$
\frac{\partial V}{\partial \tau}-\frac{\sigma^{2}}{2} \frac{\partial^{2} V}{\partial x^{2}}-\left(r-q-\frac{\sigma^{2}}{2}\right) \frac{\partial V}{\partial x}+r V=0 .
$$

Then the associated boundary condition is given by

$$
\lim _{\mathrm{n} \rightarrow-\infty} \mathrm{V}(\mathrm{x}, \tau)=0, \lim _{\mathrm{n} \rightarrow \infty} \mathrm{V}(\mathrm{x}, \tau)=e^{x}-K .
$$

Assume that the solution region is:

$$
\sum_{0}=\{-\infty \leq x<+\infty, 0 \leq \tau \leq T\} .
$$

In order to construct a two-step asymmetric difference scheme, we first discredit the region $\sum_{0}$ as an uniform grid with space step $\mathrm{h}$ and time step $\mathrm{k}$,

$$
\begin{gathered}
X_{j}=j h, j=0, \pm 1, \pm 2, \cdots N \\
\tau_{n}=n k, n=0,1,2 \cdots M(M=[T / K]) .
\end{gathered}
$$

Let $V_{j}^{n}$ denote the numerical approximation of the solution $\left(\mathrm{X}_{j}, \tau_{n}\right)$. Firstly, the time derivative $\frac{\partial V}{\partial \tau}$ at each grid point $\mathrm{V}\left(X_{j}, \tau_{n}\right)$ can be approximated by the BDF1:

$$
\frac{\partial V}{\partial \tau} \approx \frac{V_{j}^{n+1}-V_{j}^{n}}{k} .
$$

Secondary, for constructing the second order asymmetric finite difference method, the first and the second derivative for spatial variables can be approximated by :

$$
\begin{gathered}
\frac{\partial V}{\partial x} \approx \frac{V_{j+1}^{n}-V_{j}^{n}+V_{j}^{n+1}-V_{j-1}^{n+1}}{2 h}, \\
\frac{\partial^{2} V}{\partial x^{2}} \approx \frac{V_{j+1}^{n}-V_{j}^{n}-V_{j}^{n+1}+V_{j-1}^{n+1}}{h^{2}},
\end{gathered}
$$

Here $\mathrm{r} V$ is approximated by $\mathrm{r}\left(\frac{V_{j}^{n+1}+V_{j}^{n}}{2}\right)$.

Hence, (1) can be rewritten as following: 


$$
\begin{aligned}
\frac{V_{j}^{n+1}-V_{j}^{n}}{k}= & \frac{\sigma^{2}}{2} \frac{V_{j+1}^{n}-V_{j}^{n}-V_{j}^{n+1}+V_{j-1}^{n+1}}{h^{2}} \\
& +\left(r-q-\frac{\sigma^{2}}{2}\right) \frac{V_{j+1}^{n}-V_{j}^{n}+V_{j}^{n+1}-V_{j-1}^{n+1}}{2 h} \\
& -r\left(\frac{V_{j}^{n+1}+V_{j}^{n}}{2}\right) .
\end{aligned}
$$

And the above equation can be simplified as:

$$
V_{j}^{n+1}=a_{1} V_{j+1}^{n}+b_{1} V_{j}^{n}+c_{1} V_{j-1}^{n+1},
$$

where

$$
\begin{aligned}
& a_{1}=\frac{k \sigma^{2}+k h\left(r-q-\frac{\sigma^{2}}{2}\right)}{2 h^{2}+k \sigma^{2}+k h^{2} r-k h\left(r-q-\frac{\sigma^{2}}{2}\right)} \\
& b_{1}=\frac{2 h^{2}-k \sigma^{2}-k h^{2} r-k h\left(r-q-\frac{\sigma^{2}}{2}\right)}{2 h^{2}+k \sigma^{2}+k h^{2} r-k h\left(r-q-\frac{\sigma^{2}}{2}\right)} \\
& c_{1}=\frac{k \sigma^{2}-k h\left(r-q-\frac{\sigma^{2}}{2}\right)}{2 h^{2}+k \sigma^{2}+k h^{2} r-k h\left(r-q-\frac{\sigma^{2}}{2}\right)}
\end{aligned}
$$

Similarly if the first and the second derivative for spatial variable are approximated as follows

$$
\begin{gathered}
\frac{\partial V}{\partial x} \approx \frac{V_{j+1}^{n+1}-V_{j}^{n+1}+V_{j}^{n}-V_{j-1}^{n}}{2 h}, \\
\frac{\partial^{2} V}{\partial x^{2}} \approx \frac{V_{j+1}^{n+1}-V_{j}^{n+1}-V_{j}^{n}+V_{j-1}^{n}}{h^{2}},
\end{gathered}
$$

then, the form of (1) can be rewritten as:

$$
\begin{aligned}
\frac{V_{j}^{n+1}-V_{j}^{n}}{k}= & \frac{\sigma^{2}}{2} \frac{V_{j+1}^{n+1}-V_{j}^{n+1}-V_{j}^{n}+V_{j-1}^{n}}{h^{2}} \\
& +\left(r-q-\frac{\sigma^{2}}{2}\right) \frac{V_{j+1}^{n+1}-V_{j}^{n+1}+V_{j}^{n}-V_{j-1}^{n}}{2 h} \\
& -r\left(\frac{V_{j}^{n+1}+V_{j}^{n}}{2}\right) .
\end{aligned}
$$

Also the above equation can be simplified as:

$$
V_{j}^{n+1}=a_{2} V_{j-1}^{n}+b_{2} V_{j}^{n}+c_{2} V_{j+1}^{n+1},
$$

where

$$
a_{2}=\frac{k \sigma^{2}-k h\left(r-q-\frac{\sigma^{2}}{2}\right)}{2 h^{2}+k \sigma^{2}+k h^{2} r+k h\left(r-q-\frac{\sigma^{2}}{2}\right)}
$$

$$
\begin{aligned}
& b_{2}=\frac{2 h^{2}-k \sigma^{2}-k h^{2} r+k h\left(r-q-\frac{\sigma^{2}}{2}\right)}{2 h^{2}+k \sigma^{2}+k h^{2} r+k h\left(r-q-\frac{\sigma^{2}}{2}\right)} \\
& c_{2}=\frac{k \sigma^{2}+k h\left(r-q-\frac{\sigma^{2}}{2}\right)}{2 h^{2}+k \sigma^{2}+k h^{2} r+k h\left(r-q-\frac{\sigma^{2}}{2}\right)}
\end{aligned}
$$

Finally, the asymmetric scheme for the model problem is designed as follows: at each time level, the approximation $A_{j}^{n+1}$ are obtained by using the scheme (5) from left boundary and $B_{j}^{n+1}$ are calculated by scheme (7) from right boundary. Once both approximations are computed, the final approximations $V_{j}^{n+1}$ are taken with arithmetic mean of $A_{j}^{n+1}$ and $B_{j}^{n+1}$. More precisely, the proposed scheme is summarized as:

$$
\left\{\begin{array}{c}
A_{j}^{n+1}=a_{1} V_{j+1}^{n}+b_{1} V_{j}^{n}+c_{1} V_{j-1}^{n+1} \\
B_{j}^{n+1}=a_{2} V_{j-1}^{n}+b_{2} V_{j}^{n}+c_{2} V_{j+1}^{n+1} \\
V_{j}^{n+1}=\frac{A_{j}^{n+1}+B_{j}^{n+1}}{2} .
\end{array}\right.
$$

\section{Error Analysis}

Note the truncation error of the asymmetric scheme (8) is :

$$
\begin{aligned}
& \mathrm{T}(\mathrm{k}, \mathrm{h})=2\left(\frac{V_{j}^{n+1}+V_{j}^{n}}{k}\right) \\
& -\frac{\sigma^{2}}{2}\left(\frac{V_{j+1}^{n}-2 V_{j}^{n}+V_{j-1}^{n}+V_{j+1}^{n+1}-2 V_{j}^{n+1}+V_{j-1}^{n+1}}{h^{2}}\right) \\
& -\left(r-q-\frac{\sigma^{2}}{2}\right)\left(\frac{V_{j+1}^{n}-V_{j-1}^{n}}{2 h}+\frac{V_{j+1}^{n+1}-V_{j-1}^{n+1}}{2 h}\right) \\
& \quad+\mathrm{r}\left(V_{j}^{n+1}+V_{j}^{n}\right) .
\end{aligned}
$$

Applying the Taylor expansion of the term $\mathrm{T}(\mathrm{k}, \mathrm{h})$ about the point $V\left(\mathrm{X}_{j}, \tau_{n}\right)$, we have:

$$
\begin{aligned}
\mathrm{T}(\mathrm{k}, \mathrm{h})= & 2\left(\frac{\partial V}{\partial \tau}-\frac{\sigma^{2}}{2} \frac{\partial^{2} V}{\partial x^{2}}-\left(r-q-\frac{\sigma^{2}}{2}\right) \frac{\partial V}{\partial x}\right. \\
& +r V)+k \frac{\partial V}{\partial \tau}\left(\frac{\partial V}{\partial \tau}-\frac{\sigma^{2}}{2} \frac{\partial^{2} V}{\partial x^{2}}-\left(r-q-\frac{\sigma^{2}}{2}\right) \frac{\partial V}{\partial x}\right. \\
& +r V)+\mathrm{O}\left(k^{2}+\mathrm{h}^{2}\right)=\mathrm{O}\left(k^{2}+\mathrm{h}^{2}\right) .
\end{aligned}
$$

\section{Analysis of Stability and Convergence}

In this section, we will analysis the stability of the scheme (8). Let us consider the stabilized condition of (4). Denote $V_{j}^{n}=v^{n} e^{i j Q h}$, where $\mathrm{i}=\sqrt{-1}$ is the imaginary unit and $\mathrm{Q}$ is the wave number. Then (5) becomes:

$$
\mathrm{V}_{j}^{n+1}=\frac{b_{1}+a_{1} e^{i Q h}}{1-c_{1} e^{-i Q h}} V_{j}^{n}
$$


Define $G_{1}(h, k)=\frac{b_{1}+a_{1} e^{i Q h}}{1-c_{1} e^{-i Q h}}$

Based on the Von Neumann analysis, Equation (4) is stable if $\left|G_{1}(h, k)\right| \leq 1$. We define $\alpha=\left(r-q-\frac{\sigma^{2}}{2}\right)$ and $\beta=\frac{k \sigma^{2}}{2 h^{2}}$. Then the stabilized condition of (4) is given by:

$\left\{\begin{array}{c}\text { When } \alpha \leq 0,\left|G_{1}(h, k)\right| \text { is always smaller than } 1 . \\ \text { When } \alpha>0 \text {, and } 4 \beta-\frac{4 k \alpha \beta}{h}-\frac{k^{2} \alpha r}{h} \geq 0,\left|G_{1}(h, k)\right| \leq 1 .\end{array}\right.$

Similarly we use the same idea to calculate the stabilized condition of (6). Then the stability condition is shown as :

$\left\{\begin{array}{l}\text { When } \alpha \geq 0,\left|G_{1}(h, k)\right| \text { is always smaller than } 1 . \\ \text { When } \alpha<0 \text {, and } 4 \beta+\frac{4 k \alpha \beta}{h}+\frac{k^{2} \alpha r}{h} \geq 0,\left|G_{1}(h, k)\right| \leq 1 .\end{array}\right.$

In conclusion, we have:

Theorem 1. When $\alpha=0$, the scheme (8) for solving the payment of dividend Black-Scholes equation is unconditional stable; When $\alpha>0$ and $4 \beta-\frac{4 k \alpha \beta}{h}-\frac{k^{2} \alpha r}{h} \geq 0$, the scheme (8) is stable ; When $\alpha<0$ and $4 \beta+\frac{4 k \alpha \beta}{h}+\frac{k^{2} \alpha r}{h} \geq 0$, the scheme (8) is stable.

\section{Numerical Results and Conclusion}

In this section, we implement numerical simulations to price European call options, which is calculated by MATLAB R2011b.

\section{Example 1:}

Let us consider the European call option where the parameters used in the simulation are:

$$
\begin{aligned}
& S=100, K=100, T=0.5, \\
& \sigma=0.2, r=0.05, q=0.03 .
\end{aligned}
$$

The reference value for this example is 6.029529. In Tables $1 \& 2$, value denotes the European call options obtained by (8), where $\mathrm{M}$ is the number of time steps and $\mathrm{N}$ is the number of spatial steps.

Table 1. The value and error obtained by empolying the proposed method with fixed time step size and varying the number of spatial grids.

\begin{tabular}{|c|c|c|c|c|}
\hline $\mathrm{M}$ & $\mathrm{N}$ & Value & Error & Order \\
\hline 1200 & 128 & 6.069953 & 0.040424 & - \\
\hline 1200 & 256 & 6.047313 & 0.017784 & 1.185 \\
\hline 1200 & 512 & 6.026426 & 0.003103 & 2.519 \\
\hline 1200 & 1024 & 6.028804 & 0.000725 & 2.097 \\
\hline
\end{tabular}

Table 2. The value and error obtained by empolying the proposed method with fixed spatial discretization and varying the time step sizes.

\begin{tabular}{|c|c|c|c|c|}
\hline $\mathrm{N}$ & $\mathrm{M}$ & Value & Error & Order \\
\hline 1400 & 120 & 6.011806 & 0.017723 & - \\
\hline 1400 & 240 & 6.025169 & 0.004360 & 2.023 \\
\hline 1400 & 480 & 6.028496 & 0.001033 & 2.077 \\
\hline 1400 & 960 & 6.029327 & 0.000202 & 2.352 \\
\hline
\end{tabular}

As observed in Tables $1 \& 2$, the numerical results show that the proposed scheme (8) has second-order convergence.

Table 3 shows the comparison of the computation time between the second order asymmetric difference method (short for ADM) and the Crank-Nicolson method (short for C$\mathrm{N})$.

Table 3 Comparisons between the proposed method and the Crank-Nicolson method by varying the time step sizes and the number of spatial grids.

\begin{tabular}{|c|c|c|c|}
\hline \multirow{2}{*}{$\mathrm{M}$} & \multirow{2}{*}{$\mathrm{N}$} & \multicolumn{2}{|c|}{ CPU time(Sec) } \\
\cline { 3 - 4 } & 512 & 0.069040 & 0.192794 \\
\hline 200 & 1024 & 0.123246 & 2.209242 \\
\hline 400 & 2048 & 0.862435 & 14.059381 \\
\hline 800 & & \multicolumn{2}{c}{} \\
\hline
\end{tabular}

The figures of Table3 show ADM is superior to C-N.

\section{Example 2:}

Here we choose the parameters as follows.

$$
\begin{gathered}
S=90, K=90, T=1 / 12, \\
\sigma=0.2, r=0.05, q=0.03 .
\end{gathered}
$$

We fix the spatial step number with $\mathrm{N}=900$, and vary the time step number M from 1200 to 1440 . The errors of both the semi-implicit method and the second order asymmetric difference method are plotted in the figure 1. As showed in Figure, the error of the proposed method is faster decreasing than the semi-implicit difference scheme as increasing the number of time step $\mathrm{M}$. Therefore, the proposed scheme is superior to the semi-implicit method in sense of the efficiency of computational costs.

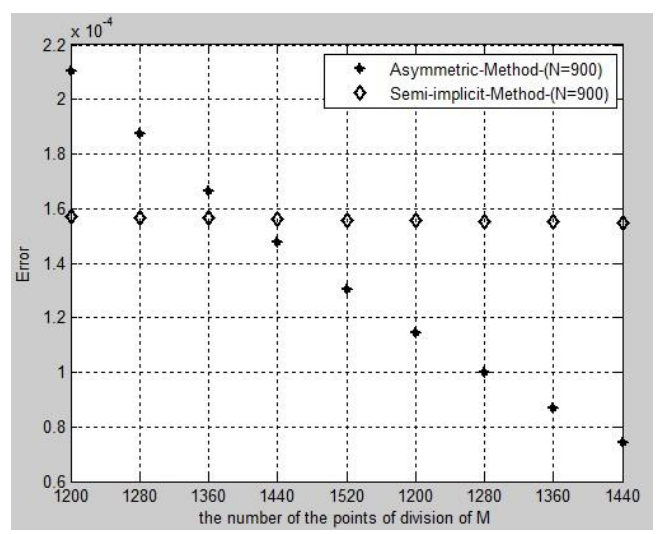

Figure 1 Error behaviors of the proposed method and the semi-implicit method with fixed $\mathrm{N}=900$ and varying $\mathrm{M}$ from 1200 to 1440 .

\section{Conclusion}

In this paper, we developed a second-order asymmetric method for solving the European call options. The accuracy of the asymmetric scheme is better than the semi-implicit scheme in most case. It also shows that the computational cost of the present method is superior to the Crank-Nicolson method. 


\section{Acknowledgment}

This work is partially supported by basic science research program through the National Research Foundation of Korea(NRF) funded by the ministry of education, science and technology (grant number 2011-0029013). The authors sincerely thank Prof. Xiaozhong Yang of North China Electric Power University for his fruitful discussions.

\section{Reference}

[1] F. Black and M. Scholes, The pricing of options and corporate liabilities, J. Political Economy, 1973(81), pp. 637-659

[2] X. Z. Yang, L. F. Wu, and W.T. Lin, Semi-implicit Difference Algorithm for Solving the Black-Scholes Equation with Payment of Dividend, Electrical \& Electronics Engineering (EEESYM) 2012 IEEE Symposium on, pp. 364-367

[3] L. F. Wu, X. Z. Yang, An Efficient Difference Algorithm for BlackScholes Equation with Payment of Dividend, Proceedings of the 2012 2nd International Conference on Computer and Information Application (ICCIA 2012), pp. 470-473

[4] F. Zhang, X.Z. Yang, A parallel difference numerical methods for solving the payment of dividend Black-Scholes equation, Sciencepaper Online, 2012, pp. 1-12
[5] Y. H. Kwon, Y. Lee, A second-order finite difference method for option pricing under jump-diffusion models, SIAM Journal on Numerical Analysis, 2011(49), pp. 2598-2617

[6] P. Brandimarte, Numerical Methods in Finance and Economics: A MATLAB-Based Introduction, -2/E, 2006

[7] D. J. Duffy, Finite Difference methods in financial engineering: a partial Differential Equation approach. John Wiley \& Sons, 2006

[8] W. Liao, A. Q. M. Khaliq, High-order compact scheme for solving nonlinear Black-Scholes equation with transaction cost, International Journal of Computer Mathematics, 2009(86), pp. 1009-1023

[9] J. Ankudinova, M. Ehrhardt, On the numerical solution of nonlinear Black-Scholes equations, Computers \& Mathematics with Applications, 2008(56), pp. 799-812

[10] L. J. Campbell, and B. Yin, On the stability of alternating-direction explicit methods for advection-diffusion equations. Numerical Methods for Partial Differential Equations, 2007(23), pp. 1429-1444

[11] H. Han, X. Wu, A Fast Numerical Method for the Black-Scholes Equation of American Options, SIAM Journal on Numerical Analysis, 2003(41), pp. 2081-2095

[12] D. Jeong, J. Kim, I. S. Wee, An accurate and efficient numerical method for Black-Scholes equations, Commun. Korean Math. Soc, 2009(24), pp. 617-628 